Culture et histoire dans l'espace roman

$6 \mid 2011$

Figures du pouvoir dans la littérature hispanoaméricaine

\title{
Pouvoir symbolique et contre-pouvoir féminin
}

La violence émancipatrice dans Cómo triunfar en la vida d'Angélica Gorodischer

\section{Maya Desmarais}

\section{CpenEdition}

\section{Journals}

Édition électronique

URL : https://journals.openedition.org/cher/9547

DOI : $10.4000 /$ cher.9547

ISSN : 2803-5992

Éditeur

Presses universitaires de Strasbourg

Édition imprimée

Date de publication : 30 juin 2011

Pagination : 175-185

ISBN : 978-2-35410-033-9

ISSN : 1968-035X

Référence électronique

Maya Desmarais, «Pouvoir symbolique et contre-pouvoir féminin », reCHERches [En ligne], 6 | 2011, mis en ligne le 17 décembre 2021, consulté le 26 janvier 2022. URL : http://journals.openedition.org/ cher/9547 ; DOl : https://doi.org/10.4000/cher.9547

\section{(c) (i) (2)(2)}

Ce(tte) œuvre est mise à disposition selon les termes de la Licence Creative Commons Attribution -

Pas d'Utilisation Commerciale - Partage dans les Mêmes Conditions 4.0 International. 


\title{
Pouvoir symbolique et contre-pouvoir féminin : la violence émancipatrice dans Cómo triunfar en la vida d'Angélica Gorodischer
}

\author{
Maya Desmarals \\ Université Toulouse II-Le Mirail \\ El Salvador (Buenos Aires)
}

e concept de pouvoir est polysémique et a fait couler beaucoup d'encre
en fonction du champ au sein duquel il est élaboré et mis en oeuvre,
pouvoir dans un rapport individuel ou collectif, assorti généralement de
violence, laquelle peut être physique ou symbolique. Nous n'appréhenderons
pas ici ce concept dans la dimension politique de gouvernance pour en
interroger la légitimité, mais choisissons plutôt de limiter l'acception du
terme "pouvoir» à l'idée de domination, une domination dite "masculine»
selon la terminologie de Pierre Bourdieu, et dont nous observerons les
réponses, c'est-à-dire la mise en œuvre du contre-pouvoir. Mais il s'agit
bien d'une domination inscrite dans une logique sociopolitique, raison pour
laquelle les réponses mettent en place diverses stratégies, tant au niveau
politique que linguistique ou artistique. Notre analyse se centrera ici sur
le choix des techniques littéraires dans le but de remettre en question ce
rapport de domination genré depuis la position périphérique du dominé,
tout particulièrement à partir de la mise en scène de la violence.
Ainsi, nous nous intéressons ici à l'écriture d'Angélica Gorodischer,
une auteure argentine contemporaine née en 1928 qui affiche ouvertement
un féminisme de l'égalité dans ses interventions publiques, mais qui réfute
toute écriture militante ou pamphlétaire; nous nous proposons d'analyser
les stratégies que cette écrivaine met en oeuvre dans le but de remettre en

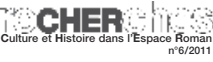


question l'ordre symbolique qui sous-tend l'organisation androcentrique de la société ${ }^{1}$.

Mais il ne s'agit en aucun cas, dans l'œuvre d'Angélica Gorodischer, d'une écriture engagée dont la thématique serait placée au service d'un discours féministe. En effet, cette auteure a choisi de développer une écriture avec une "conscience de genre $»^{2}$ qui repose sur la transcendance des catégories génériques et des codes normatifs, lesquels sont dictés par des canons, supposément objectifs et universels, mais qui constituent en réalité un outil de pouvoir placé au service de la domination. Ainsi, dans l'ouvrage qui nous intéresse ici, Cómo triunfar en la vida (1998), l'auteure se propose de remettre en question la violence, tant physique que symbolique, dont souffrent les personnes de genre féminin, par la déconstruction des types de personnages dans le cadre d’une généricité particulièrement codifiée: le récit policier, une généricités traditionnellement imprégnée de violence.

Nous allons donc observer dans un premier temps comment l'écriture met en exergue, dans Cómo triunfar en la vida, l'ordre symbolique qui soustend l'organisation de la société, et par conséquent la répartition du pouvoir en fonction du genre. Ensuite, nous analyserons les différentes stratégies développées par l'écrivaine pour remettre en question l'aspect naturalisant sur lequel repose cet ordre symbolique, et qui sert de garant à une répartition inéquitable du pouvoir dans la société.

1 Voici une définition donnée par Pierre Bourdieu de l'ordre symbolique dans le cadre de la domination masculine: "L'ordre social fonctionne comme une immense machine symbolique tendant à ratifier la domination masculine sur laquelle il est fondé: c'est la division sexuelle du travail, distribution très stricte des activités imparties à chacun des deux sexes, de leur lieu, leur moment, leurs instruments; c'est la structure de l'espace, avec l'opposition entre le lieu d'assemblée ou le marché, réservé aux hommes, et la maison, réservée aux femmes, ou, à l'intérieur de celle-ci, entre la partie masculine, avec le foyer, et la partie féminine, avec l'étable, l'eau et les végétaux; c'est la structure du temps, journée, année agraire, ou cycle de vie, avec les moments de rupture, masculins, et les longues périodes de gestation, féminines.» (Bourdieu 1998: 22-23).

2 Expression que nous préférons à celle d' "écriture féminine» trop ambiguë ou à celle d' "écriture féministe», imprégnée d'essentialisme et objet d'une connotation négative.

3 Nous préférons employer le terme de généricité à celui de genre. En effet, ce concept développé par Jean-Marie Schaeffer nous semble bien plus dynamique étant donné qu'il considère la possible évolution d'une forme générique en fonction des coordonnées spatio-temporelles et selon le régime autorial ou lectorial suivant lequel l'œuvre est appréhendée. Ce concept de généricité relève donc d'un choix actif - de la part de l'auteur ou du lecteur - qui consiste à privilégier certains traits génériques aux dépens d'autres, et à mettre ainsi en exergue une généricité plutôt qu'une autre, en fonction des traits sélectionnés et des parties de l'œuvre mises en lumière. Une forme générique peut ainsi n'être présente que dans un fragment du texte (Schaeffer 1989). 


\section{Organisation androcentique du pouvoir}

\section{La bi-partition normative}

Les situations de domination genrée assorties de discrimination sont, à l'instar de celles de race ou de classe, générées par une organisation sociale des rapports humains, dans le cadre d'un contexte historique, mais une fois passées par le prisme du discours, elles perdent leur caractère sociohistorique au profit de valeurs naturalisantes et essentialistes qui justifient a posteriori un ordre social artificiellement constitué. En d'autres termes, une construction sociale de la différence (de race, de sexe, de religion, etc.) perd son caractère historique au profit d'une explication devenue irréfutable puisqu'ahistorique, et naturelle puisqu'essentiellet. C'est ce que MarieClaude Hurtig, Michèle Kail et Hélène Rouch désignent comme «l'alibi de la "nature" ( (Hurtig, Kail, Rouch 1991: 12).

Cet alibi naturel, qui attribue une essence spécifique à chaque genre, repose sur un système d'oppositions binaires mis en évidence par Pierre Bourdieu, entre droit / courbe (et fourbe), dur / mou, dehors (public) / dedans (privé), etc., qui permet de diviser artificiellement la société en fonction de deux grandes catégories qui, à l'instar de toute généralisation, ne peuvent être que partiales et discriminantes.

Ainsi, d'après cet ordre symbolique qui divise la société en deux catégories antagonistes, en d'autres termes qui met en place une bicatégorisation sexuelle normative, ou une bipartition sexuée selon la terminologie de Nicky Le Feuvre, les hommes sont, sur le plan intellectuel, raisonnés et sages alors que les femmes sont naturellement instables, lunatiques et hystériques, et, du point de vue physique, les hommes incarnent la force et la constance alors que les femmes sont faibles et ont donc besoin de se placer sous leur protection. De même, le genre masculin, associé à la figure du père, est présenté comme autoritaire et inflexible alors que le genre féminin, de nature aimante et douce, est prédisposé à prendre soin d'autrui, tant dans le domaine familial que social. Le recours à cet ordre symbolique permet donc de légitimer le cloisonnement des rôles sociaux en fonction de ces natures distinctes, ainsi que la soumission des femmes, à partir de la survalorisation des caractéristiques considérées comme masculines.

4 Claude Zaidman met le problème en évidence lorsqu'elle considère que l'enjeu du genre comme catégorie d'analyse consiste à «remettre en cause une idéologie naturalisante liant les différences psychologiques, comportementales, sociales entre les hommes et les femmes à des différences d'ordre biologique» (Zaidman 2003: 19). 
Cette organisation naturalisante des sexes justifie l'accès des hommes aux sphères du savoir et du pouvoir, accès qui permet d'asseoir, au sein d'un cercle vicieux, la légitimité de la domination masculine.

Quelle que soit l'échelle et le type de pouvoir mis en ouvre, l'exercice $\mathrm{du}$ pouvoir repose sur la pratique de la violence, mais quelles violences entrent en jeudans le cadre de la domination masculine et comment les dénoncer dans une œuvre de fiction sans pour autant produire un pamphlet militant?

\section{Victimisation au service de la dénonciation}

La forme la plus classique de dénonciation de la violence, et la plus facile à identifier, consiste à mettre en scène des personnages de genre féminin qui subissent des violences physiques, afin de rappeler que le corps des femmes constitue un objet de lutte pour l'acquisition du pouvoir, comme c'est le cas du protagoniste de genre féminin de «Vidas privadas» qui subit une violence verbale et un début de maltraitance physique, bien qu'il ne s'agisse pas d'une violence extrême et dangereuse, comme dans Jugo de Mango où Delmira subit un enlèvement après une tentative de viol. Il s'agit davantage ici de réifier l'autre par la violence et de le/la priver de toute dignité:

- Pero ¿vos estás loca? - dijo él -. A mí no me ponés la mano, encima, ¿estamos? Asquerosa de mierda.

- ¡Me escupiste! - gritó ella -. ¡Me escupiste!

- No te merecés otra cosa - dijo él, tranquilo de nuevo.

Ella pegó uno de sus aullidos y se oyó un ruido como de cuerpo que caía. Uy, pensé, la empujó (Gorodischer 1998: 24-25).

Cela dit, exception faite de cet exemple, l'auteure n'a pas fait le choix dans cet ouvrage de la mise en scène de la violence physique, mais plutôt de la soumission, avec des personnages principalement de couches sociales inférieures, ce qui ajoute un facteur d'aliénation. Ainsi, une prostituée dans «El color de los nombres», une couturière dans «La fuente de Lerna» et une servante dans «Cómo triunfar en la vida» ont recours à la violence ou à la supercherie pour s'affranchir du rôle de soumission qui leur a été socialement imposé.

\section{Le dressage des corps}

Les nombreux travaux développés sur la violence, et tout particulièrement dans le cadre de la violence de genre, soulignent la prégnance de la violence physique dans les rapports de domination. Mais au-delà des coups portés et 
des agressions de type sexuel qui consistent à chosifier le corps de la femme et à le maintenir ainsi dans un rôle d'objet convoité, privé de subjectivité, de nombreux théoriciens à l'instar de Judith Butler, Pierre Bourdieu, Collette Guillaumin, etc. ont mis en évidence une violence moins directe mais tout aussi dangereuse qui consiste à sculpter les corps des femmes ${ }^{5}$, à les dompter afin qu'ils correspondent à un canon normé, socialement admis, associé à la représentation caricaturale du féminin par l'exacerbation des traits qui la constituent.

Ainsi, le concept de «sexage», développé par Colette Guillaumin à partir des termes «sexe » et « esclavage» ou «servage», désigne l'appropriation, non pas de la force de travail, mais plutôt du corps de la femme, appropriation placée au service de l'individu masculin ainsi que de la communauté6.

De même, Paola Tabet a observé la mise en place d'un "appareil complexe (et variable) de pression idéologique et de contrainte physique et psychique» (Tabet 1998: 94-95), dont l'objectif est le «domptage meurtrier des femmes pour en faire des corps-outils de reproduction» (Tabet 1998: 110). Ainsi, comme l'anthropologue l'a observé, alors que la création d'outils pourrait annuler la différence des potentiels de développement physique entre hommes et femmes, le monopole masculin de l'accès aux instruments a accentué cette distinction et participé à la réification du corps des femmes, assimilé lui-même à un outil.

Or, comme nous allons l'observer, Gorodischer subvertit ces codes sociaux: la protagoniste de "Una vez por semana", une jeune fille de bonne famille du début du siècle décide de se déguiser et de conduire une voiture, activité réservée alors à la gent masculine, pour acquérir le genre masculin afin de braver les interdits dus à son sexe et à sa classe, car les activités d'intérieur réservées aux femmes (piano, peinture et couture)

5 Dans une réflexion similaire mais extérieure aux distinctions de sexe, Michel Foucault a élaboré le concept de "anatomo-politiquedu corps humain " pour mettre en exergue le contrôle effectué sur le corps par la société du «bio-pouvoir». Le «dressage» du corps placé au service du capitalisme a favorisé, selon lui, «des méthodes de pouvoir susceptibles de majorer les forces, les aptitudes, la vie en général sans pour autant les rendre plus difficiles à assujettir» (Foucault 2006: 14).

6 Dans cet ouvrage, Colette Guillaumin identifie quatre modalités de cette appropriation: appropriation du temps de la femme, du produit de son corps, la mise à disposition permanente de son corps pour remplir à tout moment son devoir conjugal, ainsi que la mise à disposition de ses savoir-faire afin de prendre à sa charge la santé et le bien-être des plus fragiles (enfants, personnes âgées, malades, etc.) et des hommes valides. Colette Guillaumin présente le «sexage» comme un rapport de pouvoir, un rapport de classes, qui passe par l'appropriation matérielle de la classe féminine par la classe masculine. 
l'ennuient. Le déguisement lui permet de se libérer de l'habitus qui dicte, selon Bourdieu, usages et convenances sociales, et d'explorer les espaces extérieurs, traditionnellement réservés aux hommes.

Par ailleurs, dans «El color de los nombres», une prostituée, parangon de la chosification, est mise à disposition d'un de ses clients par sa maquerelle dans le but de l'utiliser pour dérober des bijoux.

\section{Stratégies de déconstruction de la violence symbolique?}

\section{Protagonistes de genre féminin et violence symbolique}

Angélica Gorodischer, plutôt que de dénoncer la victimisation de personnages qui souffrent de la violence de genre, s'est davantage intéressée à une forme plus subtile de violence placée au service du pouvoir, et qui est plus difficile à identifier de par sa nature: la violence dite "symbolique» (Bourdieu 1998: 54-55 et 162). Par son caractère invisible, difficilement identifiable, celle-ci «opprime» le dominé au point de lui ôter tout libre arbitre et de le mener à "collaborer» avec le dominant, si l'on reprend la terminologie de Nicole-Claude Matthieu. Ainsi, dans la nouvelle «El Beguén", la protagoniste sans nom, dévalorisée par son amant plus riche et plus puissant qui l'appelle "negrita» ou "sonsita», accepte, par amour, de sacrifier sa liberté et d'endosser le meurtre commis par celui-ci, lequel ne tardera pas à l'abandonner ${ }^{7}$. De même, la protagoniste de «La fuente de Lerna » est réduite au rôle de femme d'intérieur par son activité de couturière et à celui d'objet passif par son statut de maîtresse d'un commissaire de police, écoutant ses récits sans partager ses propres problèmes et jalousant en silence la femme de celui-ci. Ces deux femmes-outils sont placées au service du bien-être de leurs amants et ne remettent pas en question l'attitude de ces derniers, du moins au début.

\section{La violence émancipatrice : changement des rôles}

Alors que dans d'autres ouvrages de la même auteure, tels que "Cavatina» ou Jugo de Mango, la déconstruction des rôles sociaux attribués par l'ordre symbolique en fonction du sexe social s'opère à partir de la découverte de

7 Pierre Bourdieu a également consacré un paragraphe de son ouvrage La domination masculine, intitulé «Post-scriptum sur la domination et l'amour», aux relations amoureuses qui donnent, de façon illusoire, l'impression d'échapper à tout rapport de domination au nom d'un idéal utopique (148-152). 
la sensualités, dans Cómo triunfar en la vida, elle passe par la transgression des normes sociales et donc par l'appropriation des codes de la violence par les femmes.

Pour déconstruire cette prétendue nature féminine, l'auteure, en partant d'une situation traditionnelle d'hétérosexualité normative, rompt ce modèle par différents procédés discursifs, principalement par le recours à la violence dans l'ouvrage qui nous intéresse ici, une violence mise en œuvre quasi systématiquement par des protagonistes préalablement caractérisés par les caractéristiques du genre féminin: fragilité, soumission, espace intérieur, etc.

Ces actes de violence orchestrés par des protagonistes féminins servent de catalyseur afin de mettre en place des schémas d'émancipation de personnages de sexe féminin - ou de genre féminin, comme dans «Vidas Privadas » où le lecteur découvre à la fin que le protagoniste, identifié comme étant de genre féminin tout au long du récit à partir d'une perception auditive, s'avère être de sexe masculin.

Ainsi, la nouvelle «El beguén» nous dépeint les violences que les femmes s'infligent entre elles dans un univers carcéral exclusivement féminin, avec une grande cruauté et un degré de perversité qui rappelle les descriptions des tortures infligées sous la dictature par des tortionnaires masculins:

[...] te revisan entera y empieza la primera parte de la diversión. Y si llorás es peor. Primero te dan con todo y cuando te creés que ya no das más y que pronto se va a terminar, se termina, sí, pero empieza la otra parte. Si ya lo tienen todo decidido, mejor. Pero si no, una se te sienta encima, golpeada y medio desnuda como estás, sangrando por la nariz y llena de mocos y saliva y lágrimas, para que no te muevas, como si pudieras moverte, y las otras se pelean por vos. La que gana te agarra de los pelos y te lleva y te obliga a chuparla toda mientras las otras miran y se ríen y después, por ejemplo, es un decir porque se pueden hacer tantas cosas con una que recién llega, después te mete algo allá abajo entre las piernas y te hace bailar con eso colgando mientras te retorcés de dolor y las otras siguen meta risa.

Si sos buena, si te portás bien como dicen la Vaca y las otras y sobre todo las monjas que son peores que las guardias y las internas, durante un tiempo no la pasás tan mal como el primer día. Sos la sirvienta de la que te ganó, la vestís, la peinás, la bañás, le das tu comida y tu ropa y tus fasos y lo que te trae la visita, le hacés la paja, te dejás pegar y clavar agujas o aguantás que apague

8 Illustrant ainsi la théorie de Michel Foucault sur la libération de l'assujettissement à partir de la "désexualisation des plaisirs", c'est-à-dire de la découverte du plaisir en dehors du coït, et des pratiques de liberté sexuelle. Pierre Bourdieu présente également, dans La domination masculine, le rapport sexuel comme un rapport social de domination construit sur l'opposition passif/actif (37). 
los puchos apretándotelos en la barriga si hiciste algo mal o que a ella no le gustó, y ella a cambio no te da nada (Gorodischer 1998: 60-61).

Cette scène dissocie explicitement le genre féminin de la représentation féminine associée généralement à une nature maternelle empreinte de fragilité, de tendresse et de douceur. Par la suite, les rapports plus complexes de solidarité qui vont s'instaurer entre ces femmes ainsi que l'expression de souffrance de la protagoniste et les récits des sacrifices qu'elle a faits au nom de l'amour vont nuancer cette cruauté et mettre en exergue la complexité de la nature humaine, loin des schémas caricaturaux.

Mais outre sa capacité à remettre en question une représentation essentialiste caricaturale, le recours à la violence, dans une dimension émancipatrice, permet également à ces protagonistes d'acquérir une identité car elles deviennent, par cet acte fondateur, des sujets actifs. Graciela Aletta de Sylvas a d'ailleurs souligné le caractère fondateur du passage à l'acte violent pour ces femmes (Aletta de Sylvas 2009: 159).

Il peut s'agir d'un acte brutal qui permet au personnage une libération directe de la soumission, comme dans «Vidas privadas», ou d'un acte de vengeance mûrement réfléchi, comme c'est le cas dans «El beguén » et «El color de los nombres", où, suite à une trahison qui a mené les protagonistes en prison, celles-ci se vengent des années plus tard en assassinant, l'une l'amant, et l'autre le client, qui s'étaient servis d'elles. Dans «Una vez por semana", l'acte fondateur est le vol, effectué par une jeune fille de bonne famille parmi ses pairs. Lorsque la protagoniste de "La fuente de Lerna» résout l'énigme sur laquelle enquête son amant et dérobe son arme pour soutirer à une femme les bijoux volés que celle-ci détenait, il s'agit d'avantage d'une libération financière, car la condition économique constitue également un facteur d'assujettissement.

Ces actes de violence sont généralement assortis ou précédés d'une prise de conscience de la manipulation subie, comme le souligne le narrateur de «El color de los nombres» à partir de la description de la couleur des yeux - symbole universel de la conscience - de la prostituée qui est sur le point d'assassiner celui qui l'a dupée:

Todo el mundo festejó la reconciliación y el champagne avinagrado empezó a correr como río en crecida y en un segundo a nadie le importó nada pero yo vi que los ojos de la Walewska ya no estaban opacos y apagados. Ahora eran dos luces, no azul cielo como el ópalo de Negusta sino de un verde oscuro de abismo que viraba rápidamente al negro cuanto más brillaban. Sí, ella se daba cuenta (Gorodischer 1998:115). 
Par ailleurs, le choix du cadre générique du policier, et, de surcroît, du policier noir, facilite les possibilités de glissement, du rôle de victime (de violence symbolique) à celui de criminelle, ce qui, comme l'a théorisé Véronique Desnain pour qui les femmes sont les protagonistes idéales du roman noir, permet non seulement de condamner la violence de genre mais aussi de remettre en cause le concept de nature féminine.

Mais il ne s'agit pas forcément d'inverser les pôles de la domination et de rendre les femmes dominatrices ou justicières dans un nouvel ordre social qui serait construit en symétrie par rapport au nôtre, mais davantage de transcender ces rapports de domination et ces catégories artificiellement établies afin d'arriver à un nouvel ordre social dépourvu de discrimination genrée, et surtout loin de l'artifice des représentations caricaturales.

Cependant, comme il n'est jamais possible de cloisonner l'œuvre d'Angélica Gorodischer dans une généralisation taxinomique et que l'écrivaine se plaît à perturber les horizons d'attente de ses lecteurs, la nouvelle «La noche vacía» va à l'encontre de ce que nous venons d'observer, car elle présente un personnage-narrateur masculin pris parfois d'un besoin irrépressible de tuer alors qu'il ne se trouve absolument pas dans une situation de soumission. Mais l'origine de ce besoin de tuer est décrit comme un bouillonnement passionnel qu'il serait possible de rapprocher des sautes d'humeur traditionnellement associées aux femmes, à l'instar de l'hystérie: "donde hierven los sueños y los humores, los delirios" (Gorodischer 1998: 117). Il s'agit donc de présenter un personnage masculin caractérisé par des traits féminins, sans pour autant qu'il en adopte le genre, toujours dans le but de remettre en question les archétypes naturalisants. Par ailleurs, la dimension parodique est également très prégnante dans l'écriture d'Angélica Gorodischer, comme l'illustre le jeu avec les codes du policier anglais de type scientifique. Ainsi, le narrateur-assassin affirme à ses collègues médecins qu'il considère comme une ineptie la possibilité d'identifier l'assassin à partir de ses empreintes digitales.

Finalement, il s'agit moins, dans l'œuvre d'Angélica Gorodischer, et dans cet ouvrage en particulier, de proposer une forme de contre-pouvoir dans le but de "moraliser le pouvoir", que de remettre en question les fondements essentialistes qui structurent de façon arbitraire l'organisation sociale binaire. Angélica Gorodischer a donc décidé de déconstruire le discours traditionnel patriarcal - à l'origine du rapport de domination genré, désigné par Pierre Bourdieu comme la «socialisation du biologique et biologisation du social» (14) - à partir de la mise en scène de personnages, qui se trouvent 
initialement dans une situation de soumission par l'exacerbation de la violence symbolique, et qui acquièrent identité et libre arbitre par la mise en œuvre d'un acte, souvent violent, de transgression de la loi.

Puisque le discours est l'élément qui permet d'établir des hiérarchies symboliques dans l'organisation sociale et de les justifier a posteriori, c'est par le langage que tout rapport de domination commence à se mettre en place, et c'est donc par le langage que certains écrivains comme Angélica Gorodischer vont dénoncer et combattre ces rapports de domination.

Mais au-delà du travail sur les personnages, l'écrivaine place son écriture à la frontière de la post-modernité, choisissant de remettre en question toute taxinomie et de transcender les catégories génériques par des processus transgénériques qui interrogent et dynamisent le canon.

\section{Bibliographie}

Aletta de Sylvas, Graciela, 2009, La aventura de escribir. La narrativa de Angélica Gorodischer, Buenos Aires, Corregidor.

Desnain, Véronique, 2000, «Les limites de la loi: le personnage de "justicier" chez les femmes auteurs francophones contemporaines", in Féminisme et polar. Journée de l'ANEF 2000, supplément au bulletin de l'ANEF n 35, Paris, p. 55-63.

Foucault, Michel, 2006, La volonté de savoir. Droit de mort et pouvoir sur la vie, Paris, Gallimard, coll. «Folio plus Philosophie».

Guillaumin, Colette, 1992, Sexe, Race et Pratique du pouvoir: l'idée de nature, Paris, Côté-Femmes.

Gorodischer, Angélica, 1998, Cómo triunfar en la vida, Buenos Aires, Emecé.

Gorodischer, Angélica, 1988, Jugo de mango, Buenos Aires, Emecé.

Hurtig, Marie-Claude, Kail, Michèle, Rouch, Hélène (coord.), 1991, Sexe et genre. De la hiérarchie entre les sexes, Paris, CNRS Ed.

Le Feuvre, Nicky, 2003, "Le "genre" comme outil d'analyse sociologique», in Fougeyrollas-Schwebel, Dominique, Planté, Christine, Riot-Sarcey, Michèle et Zaidman, Claude (ed.), Le genre comme catégorie d'analyse. Sociologie, histoire, littérature, Paris, L'Harmattan, Bibliothèque du féminisme/ RING, p. 37-52.

Matthieu, Nicole-Claude, 1991, L'anatomie politique. Catégorisation et idéologies du sexe, Paris, Côté-femmes.

Schaeffer, Jean-Marie, 1989, Qu'est-ce qu’un genre littéraire?, Paris, Seuil, coll. «Poétique».

Tabet, Paola, 1998, La construction sociale de l'inégalité des sexes. Des outils et des corps, Paris, L'Harmattan, collection «Bibliothèque du féminisme». 
Zaidman, Claude, 2003, "Introduction» in Fougeyrollas-Schwebel, Dominique, Planté, Christine, Riot-Sarcey, Michèle et Zaidman, Claude (éd.), Le genre comme catégorie d'analyse. Sociologie, histoire, littérature, Paris, L'Harmattan, Bibliothèque du féminisme / RING, p. 9-20. 